\title{
JOURNAL OFFICIEL
}

Arrêté du 3 février 2000 fixant le calendrier des réceptions des demandes de renouvellement des autorisations relatives aux activités d'assistance médicale à la procréation et aux activités d'analyses de cytogénétique et de biologie pratiquées en vue d'établir un diagnostic prénatal in utero.

J.O. du 8 février 2000, page 1982

Article 1er . Il est ouvert une période complémentaire exceptionnelle du 15 février 2000 au 31 mai 2000 pour le dépôt des demandes tendant à obtenir en application des dispositions de l'article L.172-14 du code de la santé publique et sans modification des activités autorisées, le renouvellement des autorisations des activités d'assistance médicale à la procréation et des activités de diagnostic prénatal dont l'autorisation relève du ministre chargé de la santé en application de l'article D.712-15 dudit code, accordées en mai 1996. Les demandes sont adressées au ministre chargé de la santé sous couvert du directeur de l'agence régionale de l'hospitalisation de la région concernée.

Arrêté du 4 février 2000 fixant la composition du dossier prévu aux articles R.184-1-2 et R.673-5-2 du code de la santé publique à produire à l'appui d'une demande d'autorisation ou de renouvellement d'autorisation pour pratiquer des activités d'assistance médicale à la procréation.

J.O. du 12 février 2000 , page 2223

Art. $\mathbf{1}^{\mathbf{e r}}$. Les établissements de santé, les laboratoires d'analyse de biologie médicale et les organismes sans but lucratif désirant pratiquer une ou plusieurs des activités d'assistance médicale à la procréation définies à l'article R.152-9-1 du code de la santé publique et ceux demandant le renouvellement de leur autorisation pour ces activités doivent produire, à l'appui de leur demande, outre le dossier justificatif prévu à l'article R.172-40 du code de la santé publique, le dossier spécifique mentionné aux articles R.184-1-2 et R.673-5-2 dont le contenu est fixé au présent arrêté.

Art. 2. Le contenu du dossier concernant les activités cliniques d'assistance médicale à la procréation définies au $1^{\circ}$ de l'article R.152-9-1 du code de la santé publique est fixé à l'annexe I du présent arrêté (1).

Art. 3. Le contenu du dossier concernant les activités biologiques d'assistance médicale à la procréation définies au $2^{\circ}$ de l'article R.152-9-1 du code de la santé publique est fixé à l'annexe II du présent arrêté (1). 
Art. 4. Le dossier dont le contenu est fixé aux annexes I et II du présent arrêté doit être en outre accompagné pour chaque nouvelle demande d'agrément :

- d'un curriculum vitae du demandeur ;

- de la copie des diplômes, titres, certificats dans le domaine de la médecine ou de la biologie de la reproduction ;

- des attestations détaillées des stages effectués dans ce domaine.

Art. 5. L'ensemble du dossier est transmis par le demandeur en cinq exemplaires au directeur départemental des affaires sanitaires et sociales du département où se trouve l'établissement public de santé, le laboratoire d'analyse de biologie médicale ou l'organisme sans but lucratif.

Art. 6. L'arrêté du 2 juin 1995 fixant la composition du dossier à produire d'une demande d'autorisation de pratiquer des activités d'assistance médicale à la procréation est abrogé.

(1) L'arrêté, accompagné de l'annexe sera publié intégralement au Bulletin officiel du ministère de l'emploi et de la solidarité $n^{\circ} 2009$, disponible à la Direction des Journaux Officiels, 26, rue Desaix, 75725 Paris cedex 15, au prix de 40,40F.

\section{Arrêté du 4 février 2000 modifiant l'arrêté du 29 juin 1992 fixant la liste des sections, des sous-sections et des options ainsi que le nombre des membres de chaque sous-sec- tion des groupes du Conseil national des universités pour les disciplines médicales et odontaologiques.}

J.O. du 9 février 2000, pages 2041-2042

Art. 1. L'article $1^{\mathrm{er}}$ de l'arrêté du 29 juin 1992 susvisé est modifié ainsi qu'il suit :

I - L'intitulé de la $42^{\text {ème }}$ section : "Sciences morphologiques " est remplacé par l'intitulé suivant : «Morphologie et morphogenèse ».

II - L'intitulé de la $2^{\text {ème }}$ sous-section de la $42^{\text {ème }}$ section : “ Histologie, Embryologie, Cytogénétique » est remplacé par l’intitulé suivant : «Cytologie et histologie »

XXIII - L'intitulé de la 54 ème section : « Pathologie de l'enfant, obstétrique, système endocrinien, reproduction et développement » est remplacé par l'intitulé suivant « Développement et pathologie de l'enfant, gynécologie-obstétrique, endocrinologie et reproduction ».

XXIV - L'intitulé de la $5^{\text {ème }}$ sous-section : "Biologie du développement et de la reproduction " est remplacé par l'intitulé suivant : «Biologie et médecine du développement et de la reproduction". 